\title{
Telmisartan increases hepatic glucose production via protein kinase $C \zeta$-dependent insulin receptor substrate-1 phosphorylation in HepG 2 cells and mouse liver
}

\author{
Kae Won $\mathrm{Cho}^{1}$, Du-Hyong Cho ${ }^{2}$ \\ ${ }^{1}$ Soonchunhyang Institute of Medi-bio Science (SIMS), Soonchunhyang University, Cheonan, Korea \\ ${ }^{2}$ Department of Pharmacology, Yeungnam University College of Medicine, Daegu, Korea
}

Received: October 19, 2018

Revised: November 7, 2018

Accepted: November 7, 2018

Corresponding author:

Du-Hyong Cho

Department of Pharmacology,

Yeungnam University College of

Medicine, 170, Hyunchung-ro,

Nam-gu, Daegu 42415, Korea

Tel: +82-53-640-6974

Fax: +82-53-656-7995

Email: biohyong@hanmail.net
Background: Dysregulation of hepatic glucose production (HGP) contributes to the development of type 2 diabetes mellitus. Telmisartan, an angiotensin II type 1 receptor blocker (ARB), has various ancillary effects in addition to common blood pressure-lowering effects. The effects and mechanism of telmisartan on HGP have not been fully elucidated and, therefore, we investigated these phenomena in hyperglycemic HepG2 cells and high-fat diet (HFD)-fed mice.

Methods: Glucose production and glucose uptake were measured in HepG2 cells. Expression levels of phosphoenolpyruvate carboxykinase (PEPCK) and glucose-6-phosphatase $\alpha$ (G6Pase- $\alpha$ ), and phosphorylation levels of insulin receptor substrate-1 (IRS-1) and protein kinase $C \zeta$ (PKC) were assessed by western blot analysis. Animal studies were performed using HFD-fed mice.

Results: Telmisartan dose-dependently increased HGP, and PEPCK expression was minimally increased at a $40 \mu \mathrm{M}$ concentration without a change in G6Pase- $\alpha$ expression. In contrast, telmisartan increased phosphorylation of IRS-1 at Ser302 (p-IRS-1-Ser ${ }^{302}$ ) and decreased p-IRS-1-Tyr ${ }^{632}$ dose-dependently. Telmisartan dose-dependently increased $\mathrm{p}-\mathrm{PKC} \zeta-\mathrm{Th}^{410}$ which is known to reduce insulin action by inducing IRS-1 serine phosphorylation. Ectopic expression of dominant-negative PKC $\zeta$ significantly attenuated telmisartan-induced HGP and $\mathrm{p}-\mathrm{IRS}-1-\mathrm{Ser}^{302}$ and -inhibited $p-I R S-1-T_{y r} r^{632}$. Among ARBs, including losartan and fimasartan, only telmisartan changed IRS-1 phosphorylation and pretreatment with GW9662, a specific and irreversible peroxisome proliferator-activated receptor $\gamma$ (PPAR $\gamma$ ) antagonist, did not alter this effect. Finally, in the livers from HFD-fed mice, telmisartan increased p-IRS-1-Ser ${ }^{302}$ and decreased p-IRS-1-Tyr ${ }^{632}$, which was accompanied by an increase in $\mathrm{p}-\mathrm{PKC} \zeta-\mathrm{Thr}^{410}$.

Conclusion: These results suggest that telmisartan increases HGP by inducing $\mathrm{p}-\mathrm{PKC} \zeta-T h r^{410}$ that increases $p-I R S-1-S^{302}$ and decreases $p-I R S-1-T_{y r}{ }^{632}$ in a PPAR $\gamma$-independent manner.

Keywords: Hepatic glucose production; IRS-1; Phosphorylation; PKC乡; Telmisartan

\section{Introduction}

The anabolic hormone insulin promotes not only glucose uptake and utilization in skeletal muscles and adipose tissues but also represses glycogenolysis and gluconeogenesis in the liver that is a central organ for gluconeogenesis in the body (approximately
$90 \%$ of endogenous glucose production) $[1,2]$. Thus, hepatic dysregulation of gluconeogenesis is considered to contribute to the development of insulin resistance and type 2 diabetes mellitus $[2,3]$.

Once insulin binds to the insulin receptor (IR), the IR is autophosphorylated and activated, which mediates tyrosine 
phosphorylation of various downstream molecules including the insulin receptor substrate (IRS) $[3,4]$. Although at least six IRS isoforms (IRS-1 to -6) have been identified [4], IRS-1 has been revealed to play an important role in mediating insulin action $[5,6]$. In addition to its regulation by gene expression, IRS-1 activity is largely regulated by its extensive phosphorylation $[4,5]$. Tyrosine phosphorylation of IRS-1 is generally stimulatory (i.e., Tyr632 and Tyr896), whereas serine phosphorylation is mainly inhibitory (i.e., Ser302, Ser307, and Ser1101) $[4,5]$. Numerous kinases such as phosphatidylinositide 3-kinase (PI3K), Akt, protein kinase $\mathrm{C}(\mathrm{PKC})$, mammalian target of rapamycin, $\mathrm{p} 60$ S6 kinase, and glycogen synthase kinase-3 $\beta$ (GSK3 $\beta$ ) have been identified as responsible kinases for IRS-1 serine phosphorylation $[5,7]$. Of these, PKC-mediated serine phosphorylation of IRS-1 is widely reported to negatively regulate insulin signaling, which consequently contributes to the development of insulin resistance [7].

Telmisartan belongs to the class of angiotensin II type 1 receptor blockers (ARBs) and is widely used for the treatment of patients with hypertension with concomitant diabetes mellitus [8]. Unlike other ARBs, telmisartan exhibits various ancillary effects including improvement of insulin resistance and cardiometabolic profile [9-11]. Telmisartan is reported to improve insulin resistance in skeletal muscles via peroxisome proliferated-activated receptor $\delta$ (PPAR $\delta$ ) [12], and adipose tissues by elevating adiponectin and reducing pro-inflammatory cytokines [13]. However, the effects of telmisartan on hepatic glucose production (HGP) and its underlying mechanism have not been fully elucidated. In this study, we investigated these effects, including molecularly, using hyperglycemic HepG2 cells and high-fat diet (HFD)-fed mice, and demonstrated that telmisartan increased HGP by inducing $\mathrm{p}-\mathrm{PKC} \zeta-\mathrm{Thr}^{410}$, which increased p-IRS-1-Ser ${ }^{302}$ and decreased p-IRS-1-Tyr ${ }^{632}$ in a PPAR $\gamma$-independent manner and, consequently, impaired insulin action in HepG2 cells and the mouse liver.

\section{Materials and methods}

Telmisartan and losartan were purchased from Cayman Chemical (Ann Arbor, MI, USA). Fimasartan was a kind gift from Boryung Pharmaceutical (Seoul, Korea). D-Glucose, GW9662, sodium lactate, HEPES, dimethyl sulfoxide (DMSO), and bovine insulin were obtained from Sigma-Aldrich (St. Louis, MO, USA). Antibodies against Akt, p-Akt-Ser ${ }^{473}$, GSK3 $\beta$, p-IRS-1-Ser ${ }^{302}$, p-IRS-Ser ${ }^{307}, \mathrm{p}^{-I R S-S e r}{ }^{318}, \mathrm{p}$-IRS-Ser ${ }^{1101}, \mathrm{p}$-IRS-Tyr ${ }^{632}, \mathrm{p}$-IRS$\mathrm{Tyr}^{896}$, IRS-1, and actin were purchased from Cell Signaling Technology (Boston, MA, USA). Antibodies against glucose-6- phosphatase $\alpha$ (G6Pase- $\alpha$ ), phosphoenolpyruvate carboxykinase (PEPCK), and PKC, p-PKC $\zeta-\mathrm{Thr}^{410}$ were purchased from Santa Cruz Biotechnology (La Jolla, CA, USA). Antibodies against p-GSK3 3 -Ser ${ }^{9}$ and hemagglutinin (HA) were obtained from BD Biosciences (San Jose, CA, USA) and Covance Inc. (Emeryville, CA, USA), respectively. Low or high glucose Dulbecco's modified Eagle's medium (DMEM) and Dulbecco's phosphatebuffered saline were purchased from Welgene Inc. (Gyeongsan, Korea). Sodium pyruvate, fetal bovine serum (FBS), penicillin and streptomycin antibiotics, L-glutamine, and trypsin-EDTA solution were purchased from Gibco-BRL (Gaithersburg, MD, USA). Plasticware for cell culture was purchased from Corning Inc. (Oneonta, NY, USA) or SPL Life Sciences (Pocheon, Korea). All other chemicals were of the purest analytical grade.

\section{Cell culture and drug treatment}

The human HepG2 hepatoma cell line was a generous gift from Prof. Jiyeon Kim (Eulji University, Daejeon, Korea). HepG2 cells were maintained in high glucose $(25 \mathrm{mM})$ DMEM supplemented with $10 \% \mathrm{FBS}$ at $37^{\circ} \mathrm{C}$ in an atmosphere of $5 \% \mathrm{CO}_{2}$ in air and the cells were passaged every 3 days by trypsinization. For the experiments, HepG2 cells grown to $70 \%$ confluence were incubated in serum-free normal glucose (5.5 $\mathrm{mM}$ ) DMEM in a six-well plate overnight. After further culture in serum-free normal or high glucose DMEM containing various concentrations of telmisartan for $24 \mathrm{~h}$, the cells were cotreated with $100 \mathrm{nM}$ insulin for a further $10 \mathrm{~min}$. In some experiments, HepG2 cells were cotreated with the indicated drugs or chemicals for the specified time.

\section{Dominant negative (dn)-PKC gene transfection}

The HA-tagged rat dn-PKC $\zeta$ construct (K281R) was transfected into HepG2 cells as described in our previous report $[14,15]$ with minor modifications. Briefly, HepG2 cells were seeded in a six-well plate as described above and transfected with $1.5 \mu \mathrm{g}$ pcDNA3.1 vector containing dn-PKC $\zeta$ cDNA using Lipofectamine 2000 (Invitrogen, Carlsbad, CA, USA) according to the manufacturer's instructions. For the control, equal amounts of the pcDNA3.1 vector were transfected. After incubation for $5 \mathrm{~h}$ at $37^{\circ} \mathrm{C}$, the cells were further incubated in normal glucose DMEM containing 10\% FBS overnight before use for further experiments.

\section{Western blot analysis}

For the western blot analysis, cells treated with telmisartan in the absence or presence of various chemicals were lysed in lysis buffer (20 mM Tris-HCl pH 7.5, 150 mM NaCl, 1\% Triton X-100, 1 mM 
EDTA, 1 mM EGTA, 1 mM PMSF, $10 \mathrm{mM} \beta$-glycerophosphate, $1 \mathrm{mM} \mathrm{NaF}, 1 \mathrm{mM} \mathrm{Na}_{3} \mathrm{VO}_{4}$, and $1 \times$ protease inhibitor cocktail [Roche Molecular Biochemicals, Indianapolis, IN, USA]). In addition to HepG2 cells, the mouse livers used were dissected, and the proteins were extracted by chopping the tissue using iris scissors in ice-cold lysis buffer. The protein concentration was measured using a BCA protein assay kit (Thermo Scientific, Rockford, IL, USA). Equal quantities of protein $(20 \mu \mathrm{g})$ were electrophoretically separated on a sodium dodecyl sulfatepolyacrylamide gel and then transferred onto a nitrocellulose membrane (GE Healthcare Life Sciences, Pittsburgh, PA, USA). The blots were then probed with appropriate antibodies, followed by the corresponding secondary antibodies (Invitrogen), and finally developed using ECL reagents (Amersham Biosciences, Arlington Heights, IL, USA).

\section{Glucose uptake assay}

For glucose uptake measurements, HepG2 cells grown to $70 \%$ confluence in six-well plates were serum starved overnight and then incubated in serum-free high glucose DMEM containing various concentrations of telmisartan for $24 \mathrm{~h}$. The medium was replaced with Krebs-Ringer-phosphate-HEPES (KRPH) buffer (10 mM HEPES, pH 7.4, $136 \mathrm{mM} \mathrm{NaCl}, 4.7 \mathrm{KCl}, 5 \mathrm{mM}$ $\mathrm{KH}_{2} \mathrm{PO}_{4}, 1 \mathrm{mM} \mathrm{MgSO}_{4}, 1 \mathrm{mM} \mathrm{CaCl}$, and $2 \%$ bovine serum albumin) for glucose starvation for $40 \mathrm{~min}$. The cells were treated with $100 \mathrm{nM}$ insulin for $10 \mathrm{~min}$, and then $10 \mathrm{mM}$ 2-deoxyglucose was added to the medium for an additional $20 \mathrm{~min}$. Cell lysates were obtained, and a colorimetric glucose uptake assay was performed using a glucose uptake assay kit (Abcam, Cambridge, UK) according to the manufacturer's instructions. The uptake of glucose was normalized to the total protein content determined from the whole-cell lysates.

\section{Glucose production assay}

For the glucose production assay, HepG2 cells grown to $70 \%$ confluence were serum starved overnight, and the cells were further incubated in serum-free normal or high glucose DMEM containing various concentrations of telmisartan for $24 \mathrm{~h}$. The medium was then replaced with glucose production buffer containing $2 \mathrm{mM}$ sodium pyruvate, $20 \mathrm{mM}$ sodium lactate, and $100 \mathrm{nM}$ insulin for $4 \mathrm{~h}$. The glucose production buffer was collected, and glucose concentration was measured using a colorimetric glucose assay kit (Cayman, Ann Arbor, MI, USA) according to the manufacturer's instructions. The glucose amounts were normalized to the total protein content determined from the whole-cell lysates.

\section{Animal studies}

Male C57BL/ 6 mice were purchased from Orient Bio Inc. (Seoul, Korea) and housed in a temperature-controlled facility with a 12-h light-dark cycle. The mice were provided ad libitum access to normal chow and water until 6 weeks of age, and then they were started on an HFD (D12492, 60\% fat kcal; Research Diets Inc., New Brunswick, NJ, USA) for 13 weeks, followed by randomization to either the vehicle (DMSO)-treated $(n=5)$ or telmisartan-treated $\left(n=6 ; 5 \mathrm{mg} \cdot \mathrm{kg}\right.$ body weight ${ }^{-1} \cdot$ day $\left.^{-1}\right)$ group. Animals were treated by oral gavage and fed the HFD for an additional 5 weeks. The mice were euthanized and their livers were dissected. All the experimental procedures were performed in accordance with protocols approved by the Institutional Animal Care and Use Committee at the Soonchunhyang University (Approval no. SCH16-0002).

\section{Statistical analysis}

All results are expressed as the means \pm standard deviation (SD). Significant differences were identified using the Student's $t$-test for two points. All differences were considered significant at $p<0.05$.

\section{Results}

\section{Telmisartan increases glucose production by increasing p-IRS-1-Ser ${ }^{302}$ and decreasing p-IRS-1- $\mathrm{Tyr}^{632}$ in hyperglycemic HepG2 cells}

We first examined the effect of telmisartan on glucose production in normoglycemic or hyperglycemic HepG2 cells. In the presence of insulin treatment, telmisartan dose-dependently increased glucose production in hyperglycemic but not normoglycemic HepG2 cells (Fig. 1A). Therefore, all subsequent experiments were performed under hyperglycemic conditions. Next, we assessed the expression of G6Pase- $\alpha$ and PEPCK that are critical enzymes for hepatic gluconeogenesis. As shown in Fig. $1 \mathrm{~B}$, telmisartan at $40 \mu \mathrm{M}$ slightly increased PEPCK expression without dose-dependency, while G6Pase- $\alpha$ expression was not altered by telmisartan. Although hepatic glucose uptake is considered to be unchanged by insulin [3], glucose-induced nitric oxide has been reported to stimulate Glut4 synthesis and glucose uptake in hepatocytes [16]. Therefore, we determined whether telmisartan increased glucose uptake under our experimental conditions to rule out the possibility that glucose production may be affected by increased glucose uptake. Telmisartan at 40 $\mu \mathrm{M}$ decreased glucose uptake and Glut 4 expression but not Glut2 (Supplementary Figs. 1A, 1B), indicating that telmisartaninduced glucose production was largely caused by enhanced hepatic gluconeogenesis rather than glucose uptake. Our results 


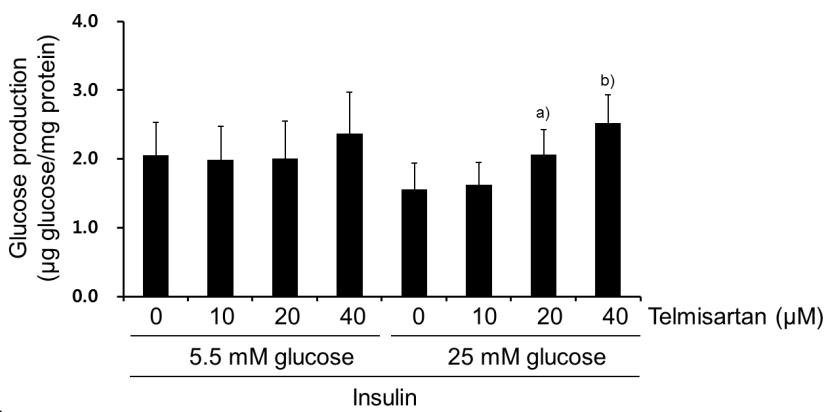

A

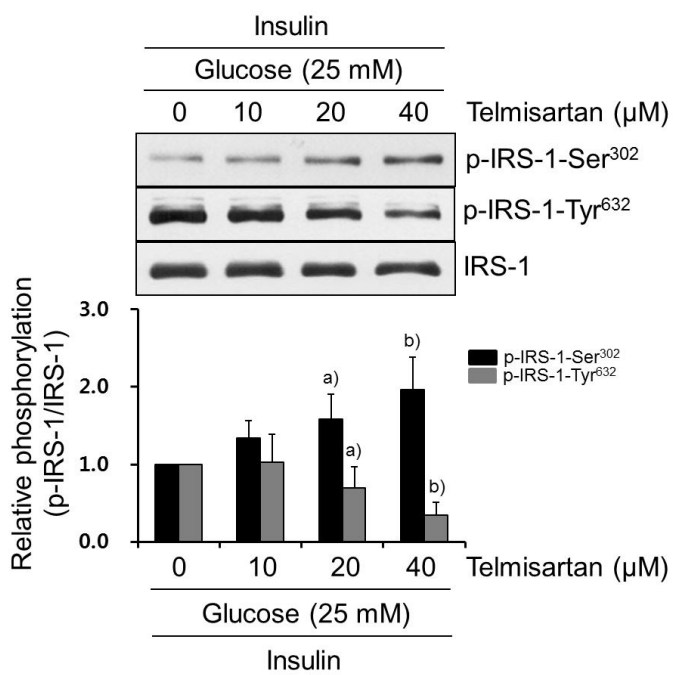

Insulin
B

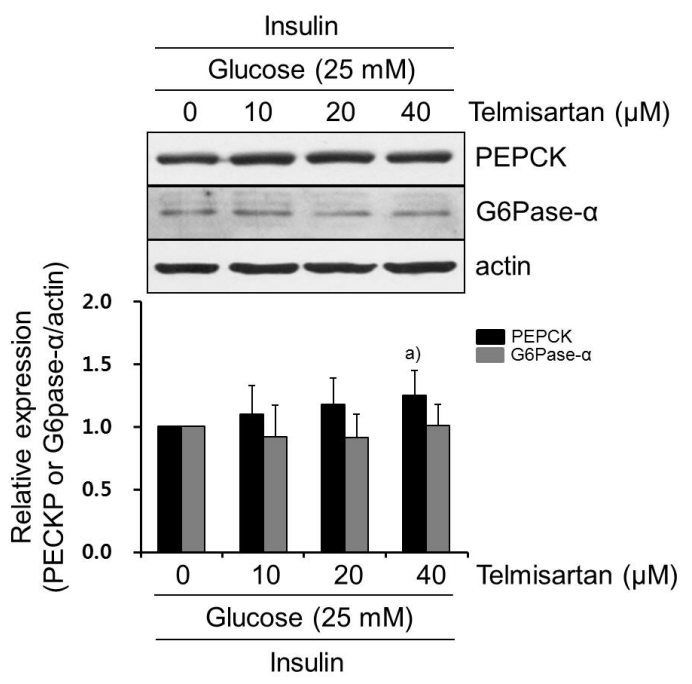

D

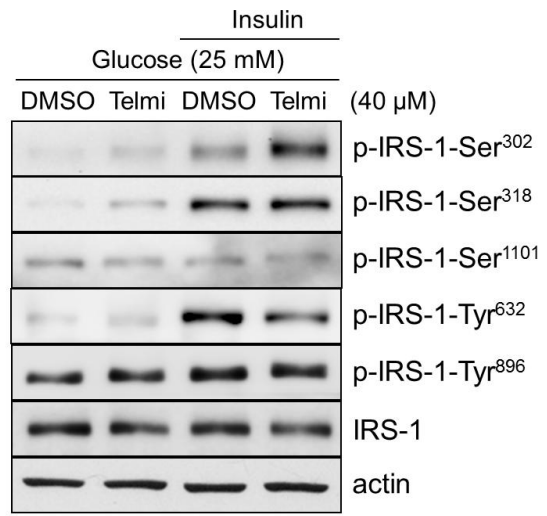

Fig. 1. Telmisartan increases glucose production by increasing $p-I R S-1-S_{e r}{ }^{302}$ and decreasing $p$-IRS-1-Tyr ${ }^{632}$ in hyperglycemic HepG2 cells. (A) HepG2 cells were treated with various doses of telmisartan $(0,10,20$, or $40 \mu \mathrm{M})$ for $24 \mathrm{~h}$ in the presence of $5.5 \mathrm{mM}$ or $25 \mathrm{mM}$ D-glucose and then glucose production assay was performed as described in the Methods. (B-D) HepG2 cells were treated with various doses of telmisartan $(0,10,20$, or $40 \mu \mathrm{M})$ for $24 \mathrm{~h}$ in the presence of $25 \mathrm{mM}$ D-glucose and then with or without $100 \mathrm{nM}$ insulin for an additional $10 \mathrm{~min}$. Total proteins were obtained, and levels of PEPCK, G6Pase- $\alpha, p-I R S-1-S_{e r}^{302}, p-I R S-1-S e{ }^{318}, p-I R S 1-S e r^{1101}, p-I R S-$ $\mathrm{Tyr}^{632}$, and $p-I R S-T y r^{896}$ were detected using western blot analysis. Nitrocellulose membranes were reprobed using antibody to detect actin or total IRS-1 to monitor equal sample loading. Densitometry was used to quantify PEPCK, G6Pase- $\alpha$, p-IRS-1-Ser ${ }^{302}$, and p-IRS-Tyr ${ }^{632}$ relative to the actin or the IRS-1 bands, respectively. Blots represent at least four experiments. Bar graphs show mean fold alterations above/below control ( $\left(\right.$ SD). Differences were statistically significant at ${ }^{\text {al }} p<0.05,{ }^{b} p<0.01$. IRS-1, insulin receptor substrate- 1 ; PEPCK, phosphoenolpyruvate carboxykinase; G6Pase- $\alpha$, glucose-6-phosphatase $\alpha$.

showed a concentration-dependent telmisartan-induced increase in glucose production but not PEPCK expression (Figs. 1A, 1B), and a previous study reported that PEPCK expression does not exclusively contribute to hepatic gluconeogenesis [17]. These observations prompted us to investigate the molecular mechanism of the telmisartan-induced HGP by first examining phosphorylation states of Akt and GSK3 $\beta$, because the increased phosphorylation of the proteins mediates insulin action [18]. Unexpectedly, telmisartan dose-dependently increased p-Akt-Ser ${ }^{473}$ and p-GSK3 3 -Ser ${ }^{9}$ (Supplementary Fig. 2), with insulin treatment. In an attempt to identify the signaling pathway mediating the effects of telmisartan, we examined the phosphorylation status of IRS-1, because it is essential and the critical molecule mediating insulin action, and IRS- 1 activity is largely regulated by its extensive phosphorylation in the presence of insulin $[4,5]$. Tyrosine phosphorylation of IRS-1 at Tyr632 and Tyr896 activates insulin action, whereas serine phosphorylation at Ser302, Ser307, and Ser1101 generally attenuates it $[4,5]$. 
In accordance with glucose production, telmisartan dosedependently increased p-IRS-1-Ser ${ }^{302}$ and decreased p-IRS$1-\mathrm{Tyr}^{632}$ with no alterations in other phosphorylation sites of IRS-1, when HepG2 cells were stimulated by insulin (Fig. 1C, Supplementary Fig. 3). Furthermore, the phosphorylation of both sites was rarely detected in the absence of insulin (Fig. 1D). These results suggest that telmisartan impaired insulin action by altering IRS-1 phosphorylation in hyperglycemic HepG2 cells.

\section{Telmisartan-induced $\mathrm{p}$-PKC $\zeta-T h r^{410}$ mediates IRS- 1 phosphorylation changes that increase glucose production}

Among various upstream kinases for IRS-1 phosphorylation, we investigated whether telmisartan affects the expression and phosphorylation of $\mathrm{PKC} \zeta$. This is because $\mathrm{PKC} \zeta$ acts as a negative feedback regulator in insulin-stimulated Fao cells and NIH-3T3 fibroblasts by decreasing tyrosine phosphorylation of IRS-1, which is secondarily caused by PKC $\zeta$-induced serine phosphorylation of IRS-1 [7,19,20]. As shown in Fig. 2A, telmisartan dose-dependently enhanced $\mathrm{p}$-PKC $\zeta-\mathrm{Thr}^{410}$ without altering its expression. This result supports that telmisartan increased PKC $\zeta$ activity because increased phosphorylation of PKC $\zeta$ at Thr410 residue located in the PKC $\zeta$ activation loop is well established to fully activate its enzymatic activity [21]. To clarify the role of PKC $\zeta$ activation by telmisartan in IRS-1 phosphorylation and glucose production, we overexpressed dnPKC $\zeta$ constructs in HepG2 cells. As shown in Figs. 2B and 2C, ectopic expression of dn-PKC $\zeta$ almost completely restored both the telmisartan-induced p-IRS-1-Ser ${ }^{302}$ and -repressed p-IRS-1$\mathrm{Tyr}^{632}$, and the telmisartan-induced glucose production.

These results clearly demonstrate that increased PKC $\zeta$ activity mediated the effects of telmisartan on IRS-1 phosphorylation and glucose production in insulin- stimulated HepG2 cells. As expected, the minimal increase in PEPCK expression induced by telmisartan was not changed by overexpression of dn-PKC $\zeta$ constructs (Fig. 2D), indicating that PEPCK expression did not affect glucose production.

\section{Among tested ARBs, only telmisartan induces p-IRS-1-Ser ${ }^{302}$ and represses $p$-IRS-1-Tyr ${ }^{632}$ via PPAR $\boldsymbol{\gamma}$-independent pathway}

We demonstrated that telmisartan increased glucose production in hyperglycemic HepG2 cells by increasing p-IRS-1-Ser ${ }^{302}$ and decreasing $\mathrm{p}-\mathrm{IRS}-1-\mathrm{Tyr}^{632}$ thereby attenuating insulin action. Although most ARBs share some structural moieties, each ARB contains highly variable side chains [9]. This paradigm led us to examine whether ARBs other than telmisartan also change IRS-1 phosphorylation.

As shown in Fig. 3A, among the ARBs tested, including losartan and fimasartan, only telmisartan increased p-IRS-1-Ser ${ }^{302}$ and decreased p-IRS-1-Tyr ${ }^{632}$, indicating that its inhibitory effects on insulin action are specific properties. Unlike other ARBs, telmisartan is also reported to act as a PPAR $\gamma$ partial agonist [9]. To investigate the role of PPAR $\gamma$ in the telmisartan-altered IRS-1 phosphorylation, we performed inhibitor studies using GW9662, a specific and irreversible PPAR $\gamma$ antagonist [22]. As shown in Fig. 3B, GW9662 did not affect telmisartan-regulated IRS-1 phosphorylation. Taken together, our results suggest that telmisartan exerts its effects on IRS-1 phosphorylation in a PPAR $\gamma$-independent manner.

\section{Telmisartan induces $p$-IRS-1-Ser ${ }^{302}$, represses p-IRS-1-Tyr ${ }^{632}$, and increases p-PKC -Thr ${ }^{410}$ in livers of HFD-fed mice}

Next, to confirm our in vitro findings in vivo, we performed animal studies by feeding mice with an HFD (60\% fat kcal) for 13 weeks (Fig. 4A). In accordance with the in vitro results, the livers from telmisartan-treated mice showed significantly higher p-IRS-1-Ser ${ }^{302}$ and lower $\mathrm{p}$-IRS-1-Tyr ${ }^{632}$ levels than those from vehicle-treated mice did (Fig. 4B), when insulin was injected through the portal vein. Furthermore, telmisartan treatment increased p-PKCY-Thr ${ }^{410}$ by $~ 2.2$-fold compared to that in vehicletreated mice (Fig. 4C). Similar to the in vitro results, PEPCK and G6Pase- $\alpha$ expression levels were slightly increased in telmisartantreated livers (Fig. 4D). However, telmisartan did not change Glut4 and Glut2 expression levels in the livers (Supplementary Fig. 4). These results revealed that the effects of telmisartan on phosphorylation of IRS-1 and PKC $\zeta$ were also evident in vivo.

\section{Discussion}

One of the most important findings in this study is that telmisartan impaired insulin action in hyperglycemic hepatocytes by increasing $\mathrm{p}$-IRS-1-Ser ${ }^{302}$ and decreasing $\mathrm{p}$-IRS-1-Tyr ${ }^{632}$, and consequently increased HGP (Figs. 1, 4). Although the effect of telmisartan on HGP has not been reported, several clinical trials reported that telmisartan reduces incidence of new-onset diabetes despite the different magnitude of the effects between trials [23]. A recent meta-analysis data revealed that telmisartan improves insulin resistance in patients with hypertension [10]. It was also reported that telmisartan has beneficial effects on insulin resistance in skeletal muscles and adipose tissues $[12,13]$. Based on the previous reports, therefore, our results suggest that the beneficial effects of telmisartan on glycemic control may be 


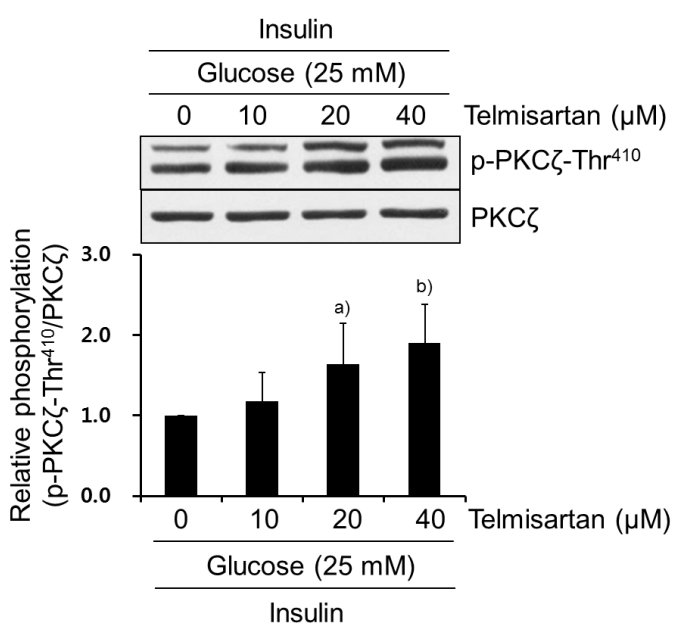

A

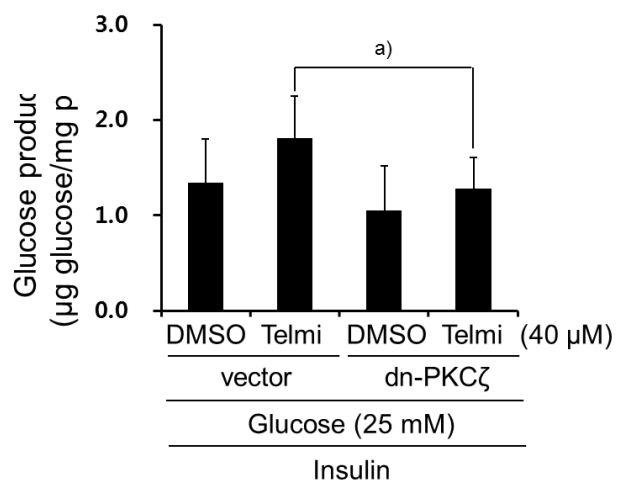

C

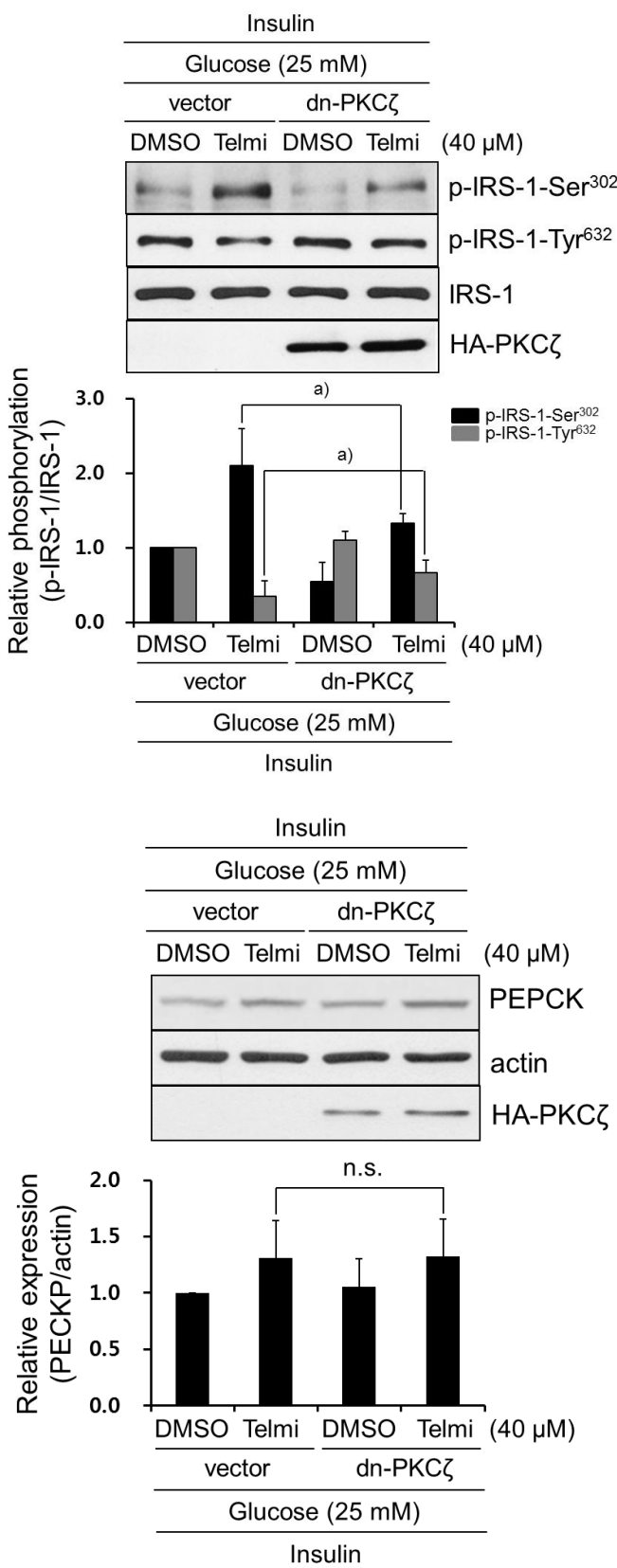

Fig. 2. Telmisartan-induced p-PKC $\zeta-T h r^{410}$ mediates IRS-1 phosphorylation changes that increase glucose production. (A) HepG2 cells were treated with various concentrations of telmisartan $(0,10,20$, or $40 \mu \mathrm{M})$ for $24 \mathrm{~h}$ in the presence of $25 \mathrm{mM}$ D-glucose and then 100 $\mathrm{nM}$ insulin for an additional $10 \mathrm{~min}$. Western blot analyses were performed as described in Fig. 1. (B) After HA-tagged dn-PKC (K281R) constructs were transfected into HepG2 cells as described in the Methods, cells were treated with $40 \mu \mathrm{M}$ telmisartan or vehicle for 24 $\mathrm{h}$ in the presence of $25 \mathrm{mM}$ D-glucose and then $100 \mathrm{nM}$ for an additional 10 min. Western blot analyses were performed as described in Fig. 1. (C) HepG2 cells were transfected with dn-PKC $\zeta$ constructs, treated with $40 \mu \mathrm{M}$ telmisartan for $24 \mathrm{~h}$ in the presence of $25 \mathrm{mM}$ D-glucose, and glucose production assay was performed as described in the Methods. (D) After transfection as described above, HepG2 cells were treated with $40 \mu \mathrm{M}$ telmisartan for $24 \mathrm{~h}$ in the presence of $25 \mathrm{mM} \mathrm{D}$-glucose and then $100 \mathrm{nM}$ insulin for an additional 10 $\mathrm{min}$. Western blot analyses were performed as described in Fig. 1. Blots represent at least four experiments. Bar graphs show mean fold alterations above/below control $\left({ }_{ \pm} \mathrm{SD}\right)$. Differences are statistically significant at ${ }^{\text {a) }} p<0.05$, ${ }^{\text {b) }} p<0.01$. n.s., not significant. PKC $\zeta_{\text {, }}$ protein kinase $C \zeta_{i}$ IRS-1, insulin receptor substrate-1; HA, hemagglutinin.

mediated by its actions on other insulin target organs such as the skeletal muscles and adipose tissues rather than the liver.
In the present study, we found that telmisartan increased HGP in hyperglycemic HepG2 cells (Fig. 1A) and, therefore, 


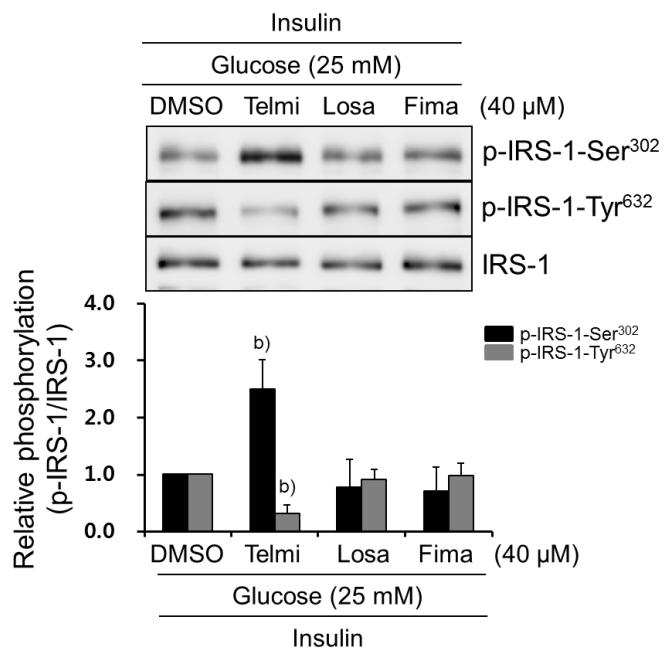

A

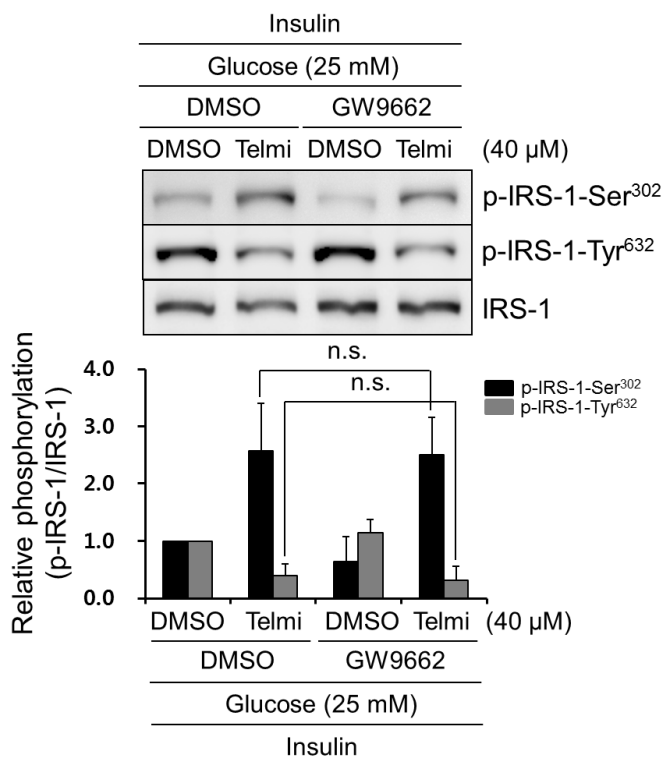

Fig. 3. Among tested ARBs, only telmisartan induces $p-I R S-1-S e r^{302}$ and represses $p$-IRS-1-Tyr ${ }^{632}$ via PPAR $\gamma$-independent pathway. (A) HepG2 cells were treated with various ARBs (telmisartan, losartan, or fimasartan at $40 \mu \mathrm{M}$ ) for $24 \mathrm{~h}$ in the presence of $25 \mathrm{mM}$ D-glucose and then $100 \mathrm{nM}$ insulin for further $10 \mathrm{~min}$. Western blot analyses were performed as described in Fig. 1. (B) After pretreatment with 5 $\mu \mathrm{M}$ GW9662 for $1 \mathrm{~h}$, HepG2 cells were treated with $40 \mu \mathrm{M}$ telmisartan for $24 \mathrm{~h}$ in the presence of $25 \mathrm{mM}$ D-glucose and then $100 \mathrm{nM}$ insulin for further $10 \mathrm{~min}$. Western blot analyses were performed as described in Fig. 1. Blots represent at least four experiments. Bar graphs show mean fold alterations above/below control $( \pm S D)$. Differences were statistically significant at ${ }^{b} p<0.01$. n.s., not significant. ARB, angiotensin II type 1 receptor blocker; IRS-1, insulin receptor substrate-1; PPAR $\gamma$, peroxisome proliferator-activated receptor $\gamma$.

we expected that telmisartan may decrease $\mathrm{p}-\mathrm{Akt}-\mathrm{Ser}^{473}$ and p-GSK3 $\beta$-Ser ${ }^{9}$, because Akt and GSK3 $\beta$ have been established as important mediators of insulin action and are used as markers for insulin resistance in vitro when the phosphorylation is suppressed [18]. However, our results revealed that telmisartan clearly enhanced p-Akt-Ser ${ }^{473}$ and p-GSK3 $\beta$-Ser ${ }^{9}$ in hyperglycemic HepG2 cells, when insulin was present (Supplementary Fig. 2). Presently, we cannot fully explain the mechanism by which telmisartan increased HGP, despite the increase of p-Akt-Ser ${ }^{473}$ and p-GSK $3 \beta-S e r{ }^{9}$. In this regard, Akt 2 plays a pivotal role in the maintenance of glucose homeostasis, whereas Akt1 is not essential for the regulation of glucose homeostasis and chronically regulates the transcription of gluconeogenic enzymes rather than that of acute glucose output in vivo $[2,24,25]$. Our results and those of the previous reports suggest that telmisartan may exert its effects on HGP via a signaling pathway mediated by other signaling molecules rather than Akt1.

Although most ARBs share some structural moieties, each ARB contains highly variable side chains [9]. In terms of structural chemistry, unlike other ARBs, telmisartan contains a carboxyl group substituted for the common tetrazole group linked to the biphenyl moiety, and two benzimidazole groups that are linked tandemly [9]. Thus, telmisartan may have various ancillary effects along with its common blood pressure-lowering effects. For example, telmisartan is reported to ameliorate vascular inflammation $[26,27]$ and to act as a PPAR $\gamma$ partial agonist [9]. Obermoser et al. have recently reported that the biphenyl moiety and the centered benzimidazole group directly linked to the biphenyl moiety are critical for PPAR $\gamma$ activation [28]. We also found that among the ARBs tested, only telmisartan increased p-IRS-1-Ser ${ }^{302}$ and decreased p-IRS-1-Tyr ${ }^{632}$ in a PPAR $\gamma$-independent manner (Fig. 3). Based on the report and our findings, we speculate that the distinctive inhibitory effects of telmisartan are attributable to its structural differences from other ARBs, and especially the second benzimidazole moiety linked to the centered one may play an important role in mediating the telmisartan's effects. However, further studies, including assessment of ARBs not examined in our study, and medicinal chemistry analyses would be needed to clarify this point.

Previously, Moeschel et al. reported that the PKCל-mediated phosphorylation of IRS-1 at Ser318 attenuates IRS-1 function in response to prolonged insulin stimulation [29]. However, other inhibitory serine residues that phosphorylated by $\mathrm{PKC} \zeta$ are not fully identified.

Furthermore, effects of the PKC $\zeta$-inhibited IRS-1 activity on its downstream effectors including PI3K and Akt are rarely 


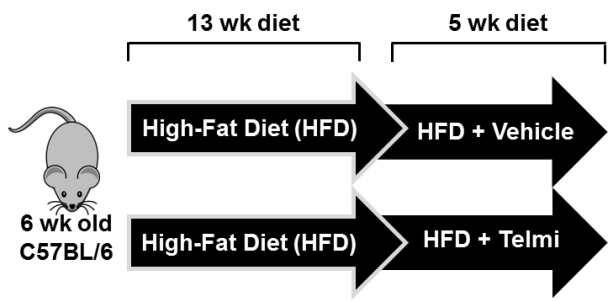

A

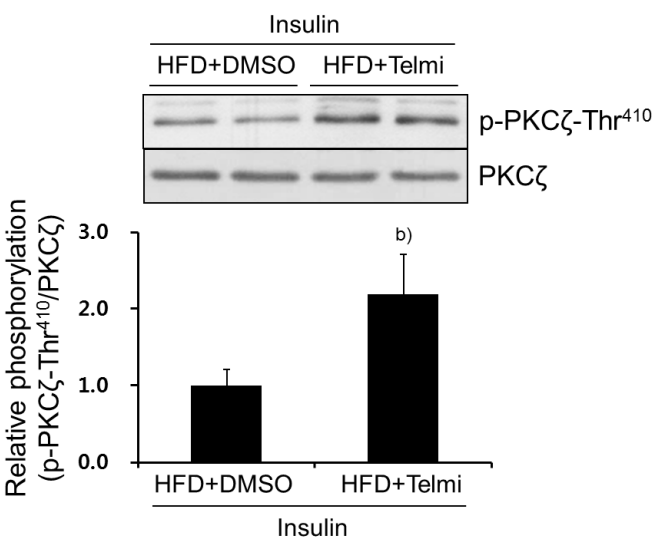

C

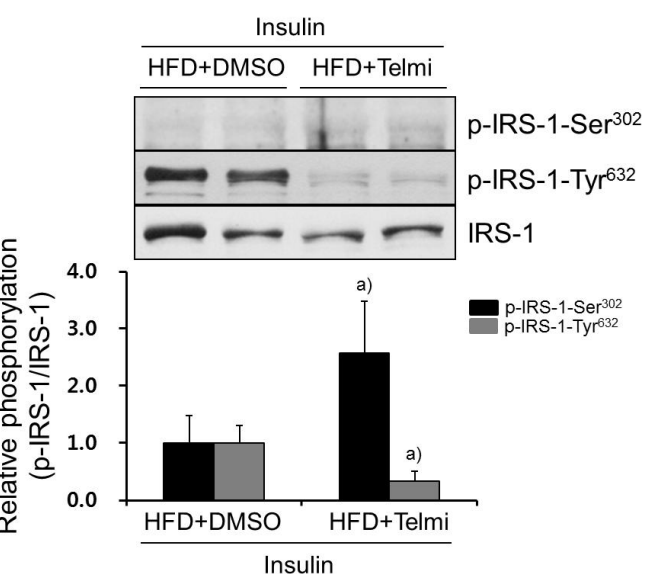

B

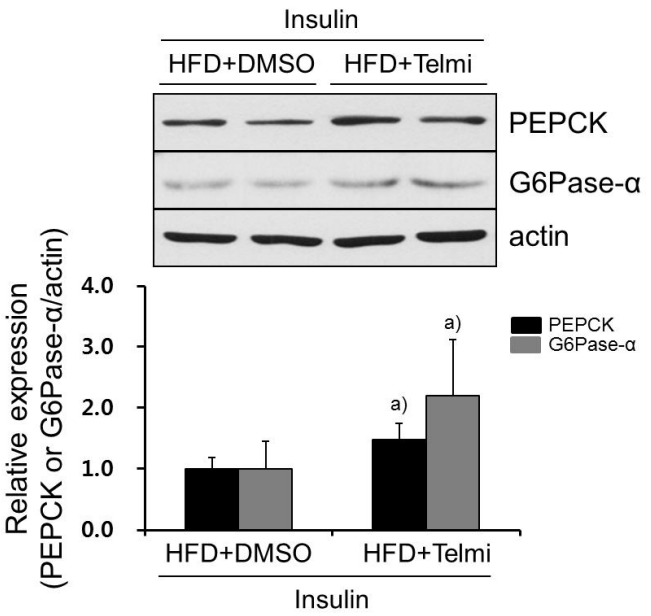

Fig. 4. Telmisartan induces $p-I R S-1-S^{302}{ }^{30}$, represses $p-I R S-1-T_{y r}{ }^{632}$, and increases $p-P K C \zeta-T h r^{410}$ in livers of HFD-fed mice. (A) Schematic diagram of animal experiments. Hyperglycemic mouse models were established as described in the Methods. (B-D) Mice were euthanized, their livers were dissected, total liver proteins were extracted, and then subjected to western blot analysis as described in Fig. 1. Blots represent at least five livers from each mouse group. Bar graphs show mean fold alterations above/below control ( \pm SD). Differences are statistically significant at ${ }^{\mathrm{a})} p<0.05,{ }^{\mathrm{b}} p<0.01$. IRS-1, insulin receptor substrate- 1 ; $\mathrm{PKC} \xi_{1}$, protein kinase $\mathrm{C} \xi_{i} \mathrm{HFD}$, high-fat diet.

elucidated, although $\mathrm{PKC} \zeta$ has been reported to be able to initiate a negative feedback regulation in the insulin-stimulated cells by decreasing tyrosine phosphorylation of IRS-1, which is secondarily caused by PKC -induced serine phosphorylation of IRS-1 $[7,19,20]$. In this respect, Ravichandran et al. revealed that overexpression of wild-type PKC $\zeta$ reduces the activity of PI3K which is associated IRS-1 without inhibition of total PI3K activity [20] and PKC $\zeta$-induced phosphorylation of IRS1 specifically interferes with the phosphorylation of IRS- 1 at Tyr612 and Tyr632 [20]. In accordance with the report, we showed that $\mathrm{PKC} \zeta$ activated by telmisartan attenuated insulin action in hyperglycemic hepatocytes by increasing p-IRS-1$\mathrm{Ser}^{302}$ and decreasing p-IRS-1-Tyr ${ }^{632}$ (Figs. 2, 4). Therefore, our results suggest that the PKC -mediated phosphorylation of IRS-1 at Ser302 plays an important role in the negative feedback regulation of IRS-1 activity. In addition, we also found that telmisartan elevated $\mathrm{p}$-Akt-Ser ${ }^{473}$ that is considered as an important downstream effector of IRS-1 and PI3K which mediate insulin signaling (Supplementary Fig. 2). Based on the report and our results, we speculate that telmisartan augmented a negative feedback signaling pathway through PKC $\zeta$ activation. Following insulin treatment, PI3K is activated and phosphorylates its downstream effectors including phosphoinositide-dependent protein kinase-1 (PDK-1) by which PDK-1 is activated and phosphorylates Akt1 and PKC . Telmisartan may have promoted PDK-1-mediated PKC $\zeta$ phosphorylation and activated PKC $\zeta$ increases p-IRS-1-Ser ${ }^{302}$, which reduces $\mathrm{p}-\mathrm{IRS}-1-\mathrm{Tyr}^{632}$. This process finally attenuates the activity of IRS-1, although activated PDK-1 could still phosphorylate Akt1, elevating p-Akt-Ser ${ }^{473}$. In support of this notion, PDK-1 is the best known upstream 
kinase for Akt1 phosphorylation, and our results showed that telmisartan increased p-Akt-Ser ${ }^{473}$ (Supplementary Fig. 2), and a previous study also reported that PDK-1 phosphorylates $\mathrm{PKC} \zeta$ at threonine 410 and activates it [21].

Previously, high interpatient variability in telmisartan plasma concentrations has been reported in patients with mild to moderate hypertension; mean $+/-\mathrm{SD}$ values for Cmax are 159 +/- $104 \mathrm{ng} / \mathrm{mL}$ for telmisartan $40 \mathrm{mg}, 693+/-606 \mathrm{ng} / \mathrm{mL}$ for telmisartan $80 \mathrm{mg}$, and $1635+/-1406 \mathrm{ng} / \mathrm{mL}$ for telmisartan 120 $\mathrm{mg}$, which are equivalent to $0.31+/-0.20 \mu \mathrm{M}$ for telmisartan 40 $\mathrm{mg}, 1.35+/-1.12 \mu \mathrm{M}$ for telmisartan $80 \mathrm{mg}$, and $3.12+/-2.73$ $\mu \mathrm{M}$ for telmisartan $120 \mathrm{mg}$, respectively [30]. Similarly, Zhang et al. have also reported that mean $+/-\mathrm{SD}$ values for Cmax in healthy Chinese subjects are $163.2+/-128.4 \mathrm{ng} / \mathrm{mL}$ and $905.7+/-583.4$ $\mathrm{ng} / \mathrm{mL}$ for $40 \mathrm{mg}$ and $80 \mathrm{mg}$ telmisartan, which are equivalent to $0.32+/-0.25 \mu \mathrm{M}$ and $1.76+/-1.13 \mu \mathrm{M}$ for $40 \mathrm{mg}$ and $80 \mathrm{mg}$ telmisartan, respectively [31]. If telmisartan concentrations used in our in vitro experiments $(10-40 \mu \mathrm{M})$ are simply compared to these clinical data, these seem to be higher. However, we clearly showed that telmisartan exerted its effects on HGP, p-IRS-1-Ser ${ }^{302}$, p-IRS-1$\mathrm{Tyr}^{632}$, and $\mathrm{p}-\mathrm{PKC} \zeta-\mathrm{Thr}^{410}$ at $20 \mu \mathrm{M}$ telmisartan, although profound effects were exhibited at $40 \mu \mathrm{M}$ telmisartan (Figs. 1A, 1C, 2A). In addition, higher concentration of telmisartan at $100 \mu \mathrm{M}$ has been used in other in vitro investigations $[32,33]$. With taking account of our results and other in vitro experiments using telmisartan, concentration of telmisartan used in the present study is considered fairly compatible with a clinically observed peak Cmax.

In conclusion, we demonstrate that telmisartan increases HGP by inducing $\mathrm{p}$-PKC $-\mathrm{Thr}^{410}$, which increases p-IRS-1-Ser ${ }^{302}$ and decreases $\mathrm{p}$-IRS-1-Tyr ${ }^{632}$ in a PPAR $\gamma$-independent manner. These actions consequently impair the action of insulin in hyperglycemic HepG2 cells and HFD-fed mouse livers. These results suggest that the beneficial effects of telmisartan on insulin resistance and cardiometabolic profile $[10,11]$ may be mediated by its actions on other insulin target organs such as the skeletal muscles and adipose tissues rather than the liver.

\section{Supplementary materials}

Supplementary materials related to this article can be found online at https://doi.org/10.12701/yujm.2019.00059

\section{Acknowledgements}

This work was supported by the National Research Foundation of Korea (NRF) grant funded by the Korea government (2015R1D1A1A01058499).

\section{Conflicts of interest}

All authors declare that no conflicts of interest exists.

\section{ORCID}

Kae Won Cho, https://orcid.org/0000-0001-7512-6722

Du-Hyong Cho, https://orcid.org/0000-0002-9891-1464

\section{References}

1. Ekberg K, Landau BR, Wajngot A, Chandramouli V, Efendic $\mathrm{S}$, Brunengraber $\mathrm{H}$, et al. Contributions by kidney and liver to glucose production in the postabsorptive state and after $60 \mathrm{~h}$ of fasting. Diabetes 1999;48:292-8.

2. Hatting M, Tavares CDJ, Sharabi K, Rines AK, Puigserver P. Insulin regulation of gluconeogenesis. Ann N Y Acad Sci 2018;1411:21-35.

3. Saltiel AR, Kahn CR. Insulin signalling and the regulation of glucose and lipid metabolism. Nature 2001;414:799-806.

4. Taniguchi CM, Emanuelli B, Kahn CR. Critical nodes in signalling pathways: insights into insulin action. Nat Rev Mol Cell Biol 2006; 7:85-96.

5. Boura-Halfon S, Zick Y. Phosphorylation of IRS proteins, insulin action, and insulin resistance. Am J Physiol Endocrinol Metab 2009;296:E581-91.

6. Tamemoto H, Kadowaki T, Tobe K, Yagi T, Sakura H, Hayakawa T, et al. Insulin resistance and growth retardation in mice lacking insulin receptor substrate-1. Nature 1994;372:182-6.

7. Schmitz-Peiffer C, Biden TJ. Protein kinase C function in muscle, liver, and beta-cells and its therapeutic implications for type 2 diabetes. Diabetes 2008;57:1774-83.

8. Andros V, Egger A, Dua U. Blood pressure goal attainment according to JNC 7 guidelines and utilization of antihypertensive drug therapy in MCO patients with type 1 or type 2 diabetes. J Manag Care Pharm 2006;12:303-9.

9. Michel MC, Foster C, Brunner HR, Liu L. A systematic comparison of the properties of clinically used angiotensin II type 1 receptor antagonists. Pharmacol Rev 2013;65:809-48.

10. Takagi H, Umemoto T; All-Literature Investigation of Cardiovascular Evidence Group. A meta-analysis of randomized trials of telmisartan versus active controls for insulin resistance in hypertensive patients. J Am Soc Hypertens 2014;8:578-92.

11. Kubik M, Chudek J, Adamczak M, Wiecek A. Telmisartan improves cardiometabolic profile in obese patients with arterial hypertension. Kidney Blood Press Res 2012;35:281-9. 
12. Li L, Luo Z, Yu H, Feng X, Wang P, Chen J, et al. Telmisartan improves insulin resistance of skeletal muscle through peroxisome proliferator-activated receptor- $\delta$ activation. Diabetes 2013;62:762-74.

13. Ushijima K, Takuma M, Ando H, Ishikawa-Kobayashi E, Nozawa M, Maekawa T, et al. Effects of telmisartan and valsartan on insulin sensitivity in obese diabetic mice. Eur J Pharmacol 2013;698:505-10.

14. Cho DH, Seo J, Park JH, Jo C, Choi YJ, Soh JW, et aI. Cyclindependent kinase 5 phosphorylates endothelial nitric oxide synthase at serine 116. Hypertension 2010;55:345-52.

15. Soh JW, Lee EH, Prywes R, Weinstein IB. Novel roles of specific isoforms of protein kinase $\mathrm{C}$ in activation of the c-fos serum response element. Mol Cell Biol 1999;19:1313-24.

16. Bhattacharya S, Ghosh R, Maiti S, Khan GA, Sinha AK. The activation by glucose of liver membrane nitric oxide synthase in the synthesis and translocation of glucose transporter-4 in the production of insulin in the mice hepatocytes. PLoS One 2013;8:e81935.

17. Burgess SC, He T, Yan Z, Lindner J, Sherry AD, Malloy CR, et al. Cytosolic phosphoenolpyruvate carboxykinase does not solely control the rate of hepatic gluconeogenesis in the intact mouse liver. Cell Metab 2007;5:313-20.

18. Zang M, Zuccollo A, Hou X, Nagata D, Walsh K, Herscovitz $\mathrm{H}$, et al. AMP-activated protein kinase is required for the lipid-lowering effect of metformin in insulin-resistant human HepG2 cells. J Biol Chem 2004;279:47898-905.

19. Liu YF, Paz K, Herschkovitz A, Alt A, Tennenbaum T, Sampson SR, et al. Insulin stimulates PKCzeta -mediated phosphorylation of insulin receptor substrate-1 (IRS-1). A self-attenuated mechanism to negatively regulate the function of IRS proteins. J Biol Chem 2001;276:14459-65.

20. Ravichandran LV, Esposito DL, Chen J, Quon MJ. Protein kinase C-zeta phosphorylates insulin receptor substrate- 1 and impairs its ability to activate phosphatidylinositol 3-kinase in response to insulin. J Biol Chem 2001;276:3543-9.

21. Bandyopadhyay G, Standaert ML, Sajan MP, Karnitz LM, Cong L, Quon MJ, et al. Dependence of insulin-stimulated glucose transporter 4 translocation on 3-phosphoinositidedependent protein kinase- 1 and its target threonine-410 in the activation loop of protein kinase C-zeta. Mol Endocrinol 1999;13:1766-72.

22. Leesnitzer LM, Parks DJ, Bledsoe RK, Cobb JE, Collins JL, Consler TG, et al. Functional consequences of cysteine modification in the ligand binding sites of peroxisome proliferator activated receptors by GW9662. Biochemistry
2002;41:6640-50.

23. Kintscher U. ONTARGET, TRANSCEND, and PRoFESS: new-onset diabetes, atrial fibrillation, and left ventricular hypertrophy. J Hypertens 2009;27(Supple 2);S36-9.

24. Cho H, Mu J, Kim JK, Thorvaldsen JL, Chu Q, Crenshaw EB 3rd, Kaestner KH, et al. Insulin resistance and a diabetes mellitus-like syndrome in mice lacking the protein kinase Akt2 (PKB beta). Science 2001;292:1728-31.

25. Cho H, Thorvaldsen JL, Chu Q, Feng F, Birnbaum MJ. Akt1/ PKBalpha is required for normal growth but dispensable for maintenance of glucose homeostasis in mice. J Biol Chem 2001;276:38349-52.

26. Song KH, Park JH, Jo I, Park JY, Seo J, Kim SA, et al. Telmisartan attenuates hyperglycemia-exacerbated VCAM-1 expression and monocytes adhesion in TNF $\alpha$-stimulated endothelial cells by inhibiting IKK $\beta$ expression. Vascul Pharmacol 2016;78:43-52.

27. Song KH, Bae SJ, Chang J, Park JH, Jo I, Cho KW, et al. Telmisartan mitigates hyperglycemia-induced vascular inflammation by increasing GSK3 $\beta$-Ser(9) phosphorylation in endothelial cells and mouse aortas. Biochem Biophys Res Commun 2017;491:903-11.

28. Obermoser V, Urban ME, Murgueitio MS, Wolber G, Kintscher U, Gust R. New telmisartan-derived PPAR $\gamma$ agonists: impact of the 3D-binding mode on the pharmacological profile. Eur J Med Chem 2016;124:138-52.

29. Moeschel K, Beck A, Weigert C, Lammers R, Kalbacher H, Voelter W, et al. Protein kinase C-zeta-induced phosphorylation of Ser318 in insulin receptor substrate-1 (IRS-1) attenuates the interaction with the insulin receptor and the tyrosine phosphorylation of IRS-1.J Biol Chem 2004;279:25157-63.

30. Smith DH, Matzek KM, Kempthorne-Rawson J. Dose response and safety of telmisartan in patients with mild to moderate hypertension. J Clin Pharmacol 2000;40:1380-90.

31. Zhang P, Zhang Y, Chen X, Li R, Yin J, Zhong D. Pharmacokinetics of telmisartan in healthy Chinese subjects after oral administration of two dosage levels. Arzneimittelforschung 2006;56:569-73.

32. Cianchetti S, Del Fiorentino A, Colognato R, Di Stefano R, Franzoni F, Pedrinelli R. Anti-inflammatory and anti-oxidant properties of telmisartan in cultured human umbilical vein endothelial cells. Atherosclerosis 2008; 198:22-8.

33. Nakano A, Hattori Y, Aoki C, Jojima T, Kasai K. Telmisartan inhibits cytokine-induced nuclear factor-kappaB activation independently of the peroxisome proliferator-activated receptor-gamma. Hypertens Res 2009;32:765-9. 